\title{
Heritable, Heterogeneous, and Costly Resistance of Sheep against Nematodes and Potential Feedbacks to Epidemiological Dynamics
}

\author{
Adam D. Hayward, ${ }_{1}^{1}$ Romain Garnier, ${ }^{2}$ Kathryn A. Watt, ${ }^{3}$ Jill G. Pilkington, ${ }^{3}$ Bryan T. Grenfell, ${ }^{2}$ \\ Jacqueline B. Matthews, ${ }^{4}$ Josephine M. Pemberton, ${ }^{3}$ Daniel H. Nussey, ${ }^{3}$ and Andrea L. Graham ${ }^{2, \dagger}$
}

1. Department of Animal and Plant Sciences, University of Sheffield, Sheffield S10 2TN, United Kingdom; 2. Department of Ecology and Evolutionary Biology, Princeton University, Princeton, New Jersey 08544; 3. Institute of Evolutionary Biology, University of Edinburgh, Edinburgh EH9 3JT, United Kingdom; 4. Moredun Research Institute, Pentlands Science Park, Bush Loan, Penicuik, Midlothian EH26 0PZ, Scotland, United Kingdom

Online enhancement: appendix.

\begin{abstract}
Infected hosts may preserve fitness by resisting parasites (reducing parasite burden) and/or tolerating them (preventing or repairing infection-induced damage). Theory predicts that these individual-level defense strategies generate divergent population-level feedbacks that would maintain genetic heterogeneity for resistance but purge heterogeneity for tolerance. Because resistance reduces parasite abundance, selection for costly resistance traits will weaken as resistance becomes common. Such negative frequency-dependent selection contrasts with predictions for tolerance, which maintains parasite abundance and so is expected to generate positive frequencydependent selection, unless, for example, tolerance trades off with resistance. Thus far, there have been few tests of this theory in natural systems. Here, we begin testing the predictions in a mammalian field system, using data on individual gastrointestinal nematode burdens, nematode-specific antibody titers (as a resistance metric), the slope of body weight on parasite burden (as a tolerance metric), and fitness from an unmanaged population of Soay sheep. We find that nematode resistance is costly to fitness and underpinned by genetic heterogeneity, and that resistance is independent of tolerance. Drawing upon empirical metrics such as developed here, future work will elucidate how resistance and tolerance feedbacks interact to generate population-scale patterns in the Soay sheep and other field systems.
\end{abstract}

Keywords: evolutionary epidemiology, costs of defense, resistance, tolerance, eco-immunology.

\section{Introduction}

Hosts interact with their parasites across many scales of biological organization. At the molecular and cellular

\footnotetext{
* This issue originated as the 2013 Vice Presidential Symposium presented at the annual meetings of the American Society of Naturalists.

$\dagger$ Corresponding author; e-mail: algraham@princeton.edu.

Am. Nat. 2014. Vol. 184, pp. S58-S76. (C) 2014 by The University of Chicago. 0003-0147/2014/184S1-55028\$15.00. All rights reserved.

DOI: $10.1086 / 676929$
}

scales, host immune systems attack parasites while parasites exploit host resources. This is the scale on which much of biomedicine focuses. However, the evolutionary fitness of both hosts and parasites is also shaped by interactions and feedbacks at much broader scales. Parasites, for example, must achieve transmission from one host organism to the next; this within-host to between-host transition is crucial to parasite life-history evolution (Frank 1996; Day et al. 2011; Mideo et al. 2011). Furthermore, population-scale ecological feedbacks are expected to affect host evolution, because the efficacy and mode of host defense exhibited in a population is expected to affect the abundance of parasites and thus the relative costs of parasitism and of defense to hosts in that population (see below). A large body of theory integrates across these biological scales to predict trajectories of hostparasite coevolution, and indeed, theory thus far outpaces empirical evidence in this area (reviewed in Boots et al. 2009; e.g., Best et al. 2009; Carval and Ferriere 2010; Debarre et al. 2012). Powerful experimental approaches can be used to test coevolutionary theories (recently reviewed by Brockhurst and Koskella (2013) and exemplified by Koskella 2014) and/or theories of how one partner will respond evolutionarily to changes in the other (e.g., Barclay et al. 2014). It is also important to test host-parasite theory in the context of realistic environmental and genetic variation in the field (Bradley and Jackson 2008; Pedersen and Babayan 2011), especially when population-scale feedbacks are hypothesized. Here, we begin to assess the assumptions and empirical predictions of theory about the evolutionary ecology of defense in a well-characterized, longitudinally monitored field system.

Hosts may alleviate the fitness costs of parasitism by 
inhibiting infection or killing parasites (in a mode of defense known as resistance) and/or by minimizing or repairing the damage associated with infection (in a mode of defense known as tolerance) (Simms 2000; Råberg et al. 2009; Baucom and de Roode 2011; Ayres and Schneider 2012; Medzhitov et al. 2012). Resistance is expected to incur physiological costs, for example, due to the huge demand for protein imposed by immune responses (Beisel 1977; Klasing 2004) and/or the potential for self-damage during an overzealous response (Graham et al. 2005). Tolerance, though mechanistically less well understood than resistance (Glass 2012), is likely to drain host resources as well, except perhaps in cases where tolerance is simply the avoidance of immunopathology (Råberg et al. 2009). Indeed, it is not yet clear whether the molecular and cellular mechanisms conferring resistance and tolerance differ (Allen and Wynn 2011; Glass 2012; Medzhitov et al. 2012), but experimental evidence for a distinction is beginning to accrue in some systems (e.g., Ferreira et al. 2011; Jamieson et al. 2013).

Theory predicts that resistance and tolerance should also have contrasting population-level consequences, via divergent effects on parasite transmission that feed back to alter selection on defense (Boots et al. 2009), as illustrated in figure 1. Resistance of individual hosts is expected to reduce parasite prevalence (percentage of hosts infected) and infection intensity (mean number of parasites per host) in the population. Resistance of individuals therefore decreases parasite transmission in the population, until transmission reaches such a low level that any costs of resistance (Beisel 1977; Klasing 2004; Graham et al. 2005) outweigh the risk of parasitism. Resistance is then expected to be selected against, and parasites can once again invade and multiply. Eventually, parasite transmission in the population becomes high enough to impose selection for resistance once more (Roy and Kirchner 2000; Miller et al. 2005). Host resistance is thus considered a negative frequency-dependent defensive trait that promotes heterogeneity, because rare genotypes with low resistance are favored when most of the host population is highly resistant and vice versa. Tolerance, by contrast, is predicted to generate positive frequency dependence: tolerance maintains host fitness without reducing parasite abundance, which enhances transmission, which sustains selection for tolerance, which enhances transmission, and so on (Roy and Kirchner 2000; Miller et al. 2005), presumably up to the physiological limits of a host (Medzhitov et al. 2012). Theory predicts fixation of tolerance alleles because, given these feedback loops, maximal tolerance should be optimal (Roy and Kirchner 2000; Miller et al. 2005). However, theory predicts that tolerance variation may also be maintained under certain conditions, for example, if a tradeoff between resistance and tolerance operates at the individual level (Restif and Koella 2003; Miller et al. 2006; Best et al. 2008), if tolerance promotes host fecundity rather than survival (Best et al. 2008), or if resistance and tolerance differ in efficacy or cost (Behnke et al. 1992; Restif and Koella 2004; Carval and Ferriere 2010). Few of these predictions have been empirically assessed. We aim to do so, in a field system that exhibits both real-world

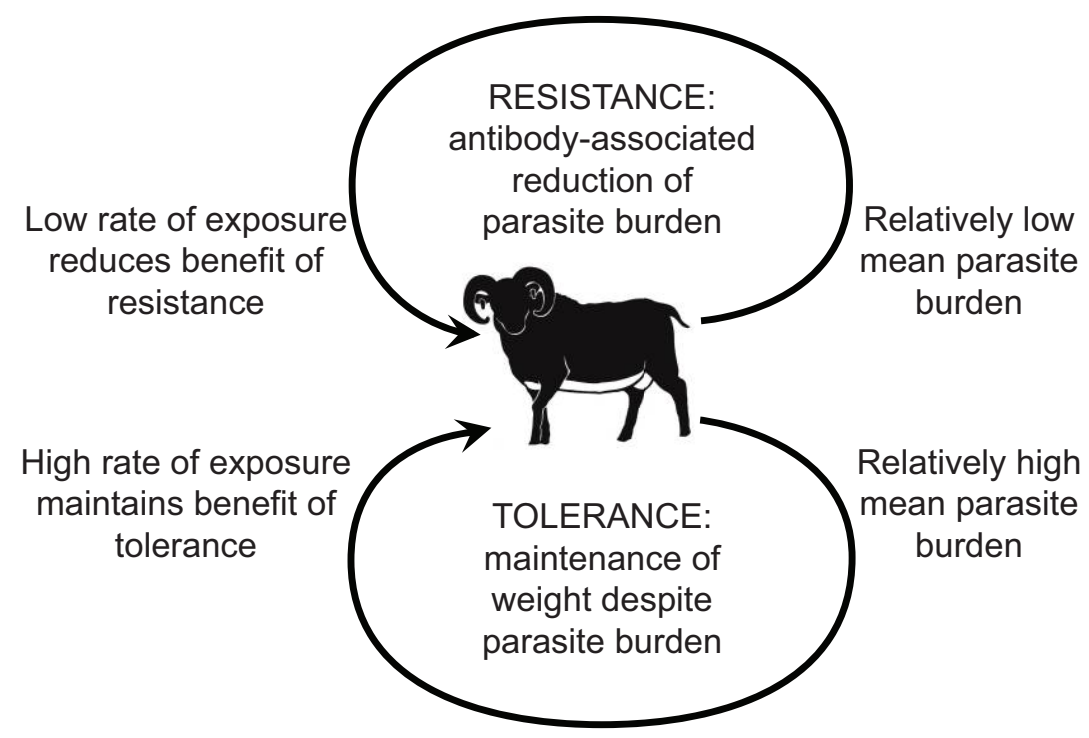

Figure 1: A summary of how individual-level defense heterogeneity (resistance vs. tolerance) is expected to generate population-level, epidemiological feedbacks that in turn alter selection on defense. Empirical metrics of resistance and tolerance used here are summarized inside the circular arrows that represent the fecal-oral strongyle nematode transmission cycle. The Soay ram was drawn by Rebecca Holland. 
complexity and the potential to link individual- and population-level processes.

The Soay sheep of the St. Kilda archipelago of Scotland present an excellent opportunity to investigate the empirical interaction of resistance and tolerance across these scales. This is because of the long-term individual-based study that has generated a plasma bank, a 9-generation pedigree, and detailed parasitological, morphometric, and demographic data (Clutton-Brock and Pemberton 2004). Parasitic strongyle nematodes, in combination with density-dependent malnutrition and poor weather in winter, are a major selective force on these sheep (Gulland 1992; Coulson et al. 2001; Wilson et al. 2004; Craig et al. 2006; Hayward et al. 2011). Adult strongyles inhabit the gastrointestinal tract and lay eggs that are released into the environment in host feces. After hatching and development, third-stage larvae (L3) migrate onto pasture and are ingested by hosts to complete the life cycle. Each Soay sheep consumes an estimated mean of 600 and maximum of 2,400 strongyle L3s per day (Gulland and Fox 1992). There is then substantial heterogeneity in the number of parasite eggs shed per sheep (i.e., fecal egg count), due to host sex (Gulland and Fox 1992), age (Craig et al. 2008; Hayward et al. 2009), behavior (Hutchings et al. 2002), and variation in genes affecting the adaptive immune response (Gulland et al. 1993; Paterson et al. 1998; Coltman et al. 2001; Beraldi et al. 2007). Together, these investigations have revealed heritability and fitness benefits of nematode resistance (estimated as the inverse of fecal egg count) but, to date, no costs of resistance (Hayward et al. 2011), on which the foregoing theory depends (Boots et al. 2009). Furthermore, the relative contributions of exposure and resistance or tolerance to observed nematode burdens are not known, and heritability of tolerance has yet to be investigated.

To address these gaps, we have begun to deploy the empirical and statistical tools necessary to quantify variation in resistance and tolerance among the Soay sheep. We report our progress to date in this study and two companion articles (Hayward et al., forthcoming; Nussey et al. 2014). The rationale for our approach across these three studies is as follows. Accompanied by data on parasite burden, host survival, and fecundity, measurements of immune phenotype often enable detection of costs of resistance that were previously obscured (Graham et al. 2011). To quantify resistance, we chose to measure the immune phenotype of nematode-specific antibody titer, because domesticated sheep use antibodies to damage and clear strongyle nematodes (e.g., Stear et al. 1995, 1997; Strain et al. 2002; Williams et al. 2010; Williams 2012), because antibodies are tractable to sample in wild animals, and because previous work showed a genetic basis for low fecal egg counts and high plasma antibody titers among
Soay lambs (Coltman et al. 2001). Having decided upon antibodies, we next had to identify the most informative specificity (e.g., against larval or adult nematodes) and functional type (e.g., immunoglobulin isotypes $\operatorname{IgA}, \operatorname{IgE}$, or IgG) to measure. As reported here, in a broad sample set of sheep (across all age classes, both sexes, and diverse population densities), we measured combined titers of all isotypes of antibodies that bound extracts of adult nematodes of an abundant species. As reported by Nussey et al. (2014), in plasma samples of ewes collected in three summers before population crashes, we separately measured titers of IgA, IgE, IgG, and IgM specific to L3 antigen extracts of the same nematode species. Together, these data sets enable assessment of heterogeneity, heritability, and potential costs as well as benefits of this metric of resistance to strongyle nematodes. Each of these characteristics is essential to the predicted negative frequency-dependence of resistance dynamics (Boots et al. 2009).

To test predictions of theory, we also needed to quantify nematode tolerance in the Soay sheep. Disease tolerance is estimated as the rate of decline in health or fitness with increasing parasite density; a flatter slope of this "reaction norm" denotes greater tolerance of a given genotype (Simms 2000; Råberg et al. 2007). With our longitudinal data, we were able to estimate the tolerance of individual sheep using random regression models to account for repeated measures while estimating variation in tolerance slopes (Hayward et al., forthcoming; Graham et al. 2011; Kause 2011). Here we use bivariate random regression to assess the relationship between resistance (i.e., nematodespecific antibody titers) and tolerance (i.e., individual reaction norms of body weight on parasite density) to determine whether Soay sheep exhibit a resistance-tolerance trade-off as assumed by much theory (Restif and Koella 2003; Miller et al. 2006; Best et al. 2008). There is precedent for such tests in wild animals (Blanchet et al. 2010), especially in common garden experiments using natural infections (e.g., Rohr et al. 2010; Tobler and Schmidt 2010; Adelman et al. 2013), but they remain rare.

In summary, to frame a test of evolutionary epidemiological theory in this natural system (with cross-scale predictions and associated empirical measures summarized in fig. 1), here we investigate (i) whether high titer of nematode-specific antibody is associated with low strongyle fecal egg count, as expected for a resistance marker; (ii) the extent of individual and genetic variation in antibody titer; (iii) whether antibody titer is positively associated with survival (suggesting a benefit of resistance) and negatively associated with reproduction (suggesting a cost of resistance); and (iv) whether tolerance and resistance trade off at the individual level. The results, together with our companion studies of ewe survival (Nussey et al. 2014) and associations of tolerance with lifetime breeding 
success (Hayward et al., forthcoming) lay the groundwork for understanding how resistance and tolerance might interact at the individual and population levels in the Soay sheep.

\section{Methods}

\section{Study Population and Data Collection}

Field Sampling. The Soay sheep (Ovis aries) inhabiting the Village Bay area of the island of Hirta, in the St. Kilda archipelago of the Outer Hebrides, Scotland, have been unmanaged for several thousand years and have been the subject of a longitudinal, individual-based demographic, genetic, and phenotypic study since 1985 (Clutton-Brock and Pemberton 2004). The majority of lambs are born in April, and $\sim 95 \%$ are captured within a week of birth and are given identification tags, have blood sampled, and are weighed. Each August, 50\% of the population is captured, weighed (to the nearest $0.1 \mathrm{~kg}$ ), and sampled for feces and blood. Fecal samples are stored at $4^{\circ} \mathrm{C}$ until parasitological examination. Blood samples are stored at $4^{\circ} \mathrm{C}$ and, within $24 \mathrm{~h}$ of collection, centrifuged at 3,000 rpm for $15 \mathrm{~min}$. Plasma is collected and stored at $-20^{\circ} \mathrm{C}$ until assayed. Population censuses are performed 10 times in each of three field seasons, providing an accurate estimate of population size. The population dynamics are characterized by large fluctuations in density, with a combination of adverse winter weather, malnutrition, and parasites contributing to periodic high-mortality winters known as "crashes" (Gulland 1992; Coulson et al. 2001; Wilson et al. 2004; Craig et al. 2006; Hayward et al. 2011).

Population Pedigree. A population pedigree has been determined through observation and molecular data (Morrissey et al. 2012). Maternities were assigned on the basis of behavioral interactions between ewes and lambs and paternities were assigned using microsatellite and allozyme marker data. Genotypic data, as well as predictors of paternity success (age and horn type) were used to assign paternity using the $\mathrm{R}$ package MasterBayes (Hadfield et al. 2006), which produces posterior distributions for both genotypic and phenotypic effects. Paternity was assigned where a candidate sire (from a pool of genotyped, tagged males) was specified in at least $80 \%$ of the samples of the posterior distributions of the pedigree, generating 2,253 assignments of paternity with mean posterior support of around 98\% (Morrissey et al. 2012). Here, we pruned the pedigree to contain only links informative to the individuals for which we had phenotypic data. The pruned pedigree contained 1,752 individuals, 1,346 with known maternities, 1,063 with known paternities, and 286 founders, with a maximum pedigree depth of 9 generations (Morrissey and Wilson 2010).

Host Body Weight and Fecal Excretion of Parasite Eggs. Body weight in August has a Gaussian distribution and is positively associated with almost every measure of Soay sheep fitness, including survival (Milner et al. 1999; Jones et al. 2005) and reproductive success (Clutton-Brock et al. 1996; Preston et al. 2003). Weight is negatively associated with measures of parasite burden in the Soay sheep (Coltman et al. 2001; Craig et al. 2008), although it is not significantly associated with tolerance of strongyles at the individual level (Hayward et al., forthcoming). These associations remain when age and leg length are taken into account. Finally, weight also serves as an approximate general measure of body condition, health, and productivity in domesticated sheep (Jackson et al. 2009; Piedrafita et al. 2010).

The trichostrongyle nematode species Trichostrongylus axei, Trichostrongylus vitrinus, and Teladorsagia circumcincta are prevalent and abundant in the Soay sheep population (Wilson et al. 2004; Craig et al. 2006). Trichostrongylus circumcincta attains $100 \%$ prevalence across all age classes and constitutes $36 \%-78 \%$ of the total nematode fauna (depending on the year and the age and sex of the host) in sheep dying over winter (Gulland 1992; Craig et al. 2006). Fecal density of the eggs of these parasites is quantified via a modification of the McMaster technique (MAFF 1986). In brief, $3 \mathrm{~g}$ of sample are homogenized with a pestle and strainer in $87 \mathrm{~mL}$ of saturated salt solution. Residual fluid is drained, and the solids are mixed and pipetted into both chambers of a $0.30-\mathrm{mL}$ McMaster slide. Strongyle nematode eggs are counted at $\times 10$ magnification, and the count is multiplied by 100 to estimate fecal egg count (often abbreviated as FEC) per gram of sample. Logistical constraints generate variation in the dry weight of samples and the interval between sampling and examination, so fecal egg count data contain more "noise" than is ideal. Nonetheless, fecal egg count is positively and linearly correlated with burden of adult nematodes counted postmortem in this population (Gulland 1992; Wilson et al. 2004) and is also a reliable indicator trait in selective breeding for parasite resistance in domestic sheep (Gruner et al. 2004; Sayers and Sweeney 2005).

Antibodies Binding Strongyle Antigens. As a putative marker of individual resistance against strongyle nematodes, here we chose to measure total immunoglobulin specific to adult $T$. circumcincta antigen (or anti-T. circumcincta antibody titer) in blood plasma samples collected in the Augusts of 1997-2007, the same sample set in which we previously measured self-reactive antibody (Graham et al. 2010). For a subset of these (from adult 
females in the summer before population "crashes" over the winters of 1998-99, 2001-02 and 2004-05), we have separately measured antibody isotypes IgA, IgE, IgG, and IgM specific to T. circumcincta larval antigens (Nussey et al. 2014). Antibodies against $T$. circumcincta may reflect host immune responses to trichostrongyle nematodes in general: in domesticated sheep, titers of T. circumcinctaspecific antibodies are positively correlated with titers of Trichostrongylus colubriformis-specific antibody (Bisset and Morris 1996; Williams et al. 2010). This suggests that antibodies specific to one species may be indicative of the strength of response to others and/or that there is crossreactivity between species. The latter is supported by the finding that only one amino acid in the sequence of the antigen tropomyosin, the recognition of which is important in protective immunity, differs between T. circumcincta and T. colubriformis (Murphy et al. 2010). Similarly, the immunogenic carbohydrate larval surface antigen is present on the surface of all ovine trichostrongylid L3 nematodes (Shaw et al. 2012). We found a positive correlation between titers of antibody binding L3 and adult T. circumcincta antigens $(r=0.80 ; N=162$; K. A. Watt, K. M. Morriss, J. B. Matthews, and A. L. Graham, unpublished data), and others have shown similar crossreactivity among life stages (e.g., Williams et al. 2010). Furthermore, our pan-isotype antibody concentrations are positively correlated with concentrations of each distinct isotype that we measured (table A1; tables A1-A5 available online). Thus, our pan-isotype anti-T. circumcincta metric arguably integrates overall antistrongyle effector activity occurring at mucosal sites (by IgA), via activated granulocytes (by IgE), and through memory (indicated by IgG; Stear et al. 1995, 1997; Strain et al. 2002; Williams et al. 2010; Williams 2012).

Antibody concentrations were measured via sandwich enzyme linked immunosorbent assays, as follows. NUNCimmuno plates (Maxisorp, Thermo Fisher Scientific) were coated with $50 \mu \mathrm{L}$ per well of crude adult T. circumcincta antigen at a concentration of $2 \mu \mathrm{g} \mathrm{mL}^{-1}$ in carbonate buffer and incubated overnight at $4^{\circ} \mathrm{C}$. Plates were emptied and then blocked with $200 \mu \mathrm{L}$ of $5 \%$ bovine serum albumin in carbonate buffer for $2 \mathrm{~h}$ at $37^{\circ} \mathrm{C}$. At this stage and after each of the following incubations, plates were washed five times with Tris-buffered saline plus 1\% Tween (TBST) using an automated plate washer. Samples were added and titrated in a 12-well doubling dilution series, ranging from $1: 50$ to $1: 1,024,000$. Plasma from a negative control (an individual of the Blackface domesticated breed that had not been exposed to parasites) was also included on each plate. Plates were then incubated for $2 \mathrm{~h}$ at $37^{\circ} \mathrm{C}$. Detection antibody (polyclonal rabbit anti-sheep Immunoglobulins/ horseradish peroxidase; DakoCytomation P0163) was added $(1: 8,000)$ at $100 \mu \mathrm{L}$ per well. Plates were incubated for $1 \mathrm{~h}$ at $37^{\circ} \mathrm{C}$ and washed 5 times with TBST and twice with distilled water. TMB substrate was then added at 100 $\mu \mathrm{L}$ per well. After $3 \mathrm{~min}$ of development, reactions were stopped with $100 \mu \mathrm{L}$ of $1 \mathrm{M} \mathrm{HCl}$. Optical density (O.D.) was measured at $450 \mathrm{~nm}$ on an Emax Precision Microplate Reader (MDS Analytical Technologies). For each assay date, the mean $+3 \mathrm{SD}$ of the O.D. of the negative control at $1: 200$ was calculated across plates. This value served as the cutoff for titer determinations for that assay date: the antibody titer for each Soay sheep sample was defined as the reciprocal of the last dilution at which binding (measured by O.D.) was $\geq 3 \mathrm{SD}$ greater than the assay datespecific mean negative control. Titer data were normalized via natural log transformation but remained discontinuous because of the discrete dilution factors of the titration. A total of 2,628 plasma samples were assayed, but some were removed from the data set because parasitological or weight data were not collected, because individuals were of unknown age or parentage or because of an unsatisfactory antibody assay (i.e., failure of the sample to generate a smooth decline in O.D. with decreasing plasma concentration; $N=43$ ). This left 2,314 observations of timepoint-matched antibody concentrations, fecal egg counts, and weights from each of 1,348 individuals. This is the sample size for analyses described below, unless otherwise indicated.

\section{Statistical Analyses}

Sheep and Parasite Dynamics. We began with exploration of population-level sheep-nematode dynamics across the entire study period. We calculated mean and standard error of August strongyle fecal egg count in lambs born each year, 1988-2012, to estimate interannual variation in the intensity of strongyle transmission. Lambs are suitable sentinels for interannual variation because they host only parasites acquired between April and August of their year of birth. We assessed the relationship between annual estimates of parasite abundance and host density using Pearson's correlation coefficient in the Hmisc package in $R$ 2.15.1.

Associations between Antibody Titer and Parasite Burden. In the 1997-2007 individual-level data set, we investigated associations between anti-T. circumcincta antibody titer and fecal egg count using linear mixed-effects models via the lme4 R package (Bates et al. 2012). We fitted untransformed fecal egg count as a fixed covariate in models of antibody titer, with fixed effects of age and sex class. We chose to focus on our new titer metric as the response variable because we wished to characterize how titer covaried with other host traits while detecting any nonlinear associations with fecal egg count. We also fitted random 
effects of individual identity, year of measurement, maternal identity, and antibody assay date to account for repeated measures and variance across these scales. We compared models incorporating linear, quadratic, and cubic functions of fecal egg count and interactions among all fixed effects. A variable was deemed to improve model fit if it affected the Akaike Information Criterion by $>2$ (Burnham and Anderson 2002) and significantly improved $\log$ likelihood $(\log \mathrm{L})$ in likelihood ratio tests, with the test statistic calculated as $-2 *\left(\log \mathrm{L}_{\text {model } 2}-\log \mathrm{L}_{\text {model1 } 1}\right)$. The significance of the change in $\log \mathrm{L}$ due to dropping a fixed effect was assessed by calculating $P$ values based on the $\chi^{2}$ statistic on the appropriate degrees of freedom.

Individual and Genetic Variation in Antibody Titer and Body Weight. We next estimated the contribution of additive genetic effects to the phenotypic variance in antibody titer and weight (in the restricted 1997-2007 data set, to complement previous genetic analyses of August weight during other portions of the time series [e.g., Coltman et al. 2001; Robinson et al. 2009]). We used quantitative genetic "animal models" implemented in ASReml 3.0 (Gilmour et al. 2009). Animal models are linear mixed-effects models that use information from the population pedigree to estimate the contribution of relatedness to phenotypic variance (Henderson 1950; Kruuk 2004; Wilson et al. 2010). For both traits, we fitted age and sex as fixed effects plus variance components (random effects), including additive genetic $\left(V_{\mathrm{A}}\right)$; permanent environment $\left(V_{\mathrm{PE}}\right)$, which accounts for nongenetic differences between individuals estimated from repeated measures across individuals; year $\left(V_{\mathrm{Y}}\right)$; maternal identity $\left(V_{\mathrm{M}}\right)$; and residual $\left(V_{\mathrm{R}}\right)$. We also fitted assay date $\left(V_{\mathrm{DATE}}\right)$ in models of antibody titer. The heritability of each trait was estimated by dividing the additive genetic variance by the total phenotypic variance $\left(h^{2}=V_{\mathrm{A}} / V_{\mathrm{P}}\right)$. The significance of variance components was assessed with likelihood ratio tests, as above.

Individual Variation in Associations between Antibody Titer, Parasite Burden, and Age. We next tested for individual and genetic variance in changes in antibody titer with increasing fecal egg count across the life span, using random regression models, in which a random effect of individual interacts with predictors such as parasite burden or age. Such models are recommended as best practice for studying phenotypic plasticity (Nussey et al. 2007) and genotype-by-environment interactions (e.g., Barton and Turelli 1989; Charmantier and Garant 2005; Kruuk et al. 2008) including tolerance (Graham et al. 2011; Kause 2011; Doeschl-Wilson et al. 2012; Kause et al. 2012), although they often demand large sample sizes (Martin et al. 2011; van de Pol 2012). We included the same fixed and random main effects as above but then allowed slopes to vary be- tween individuals by fitting an interaction between individual identity and fecal egg count in the random effects compartment of the model. Previous studies of the population have shown that additive genetic variance and heritability of traits can change with age (e.g., Wilson et al. 2007), so we also tested for individual variation in the change in antibody titer with age by fitting a randomeffect interaction between individual identity and age. For these analyses, both fecal egg count and age were standardized to zero mean and unit variance. We tested the significance of random slope terms by using likelihood ratio tests to compare results against models where individual variance in antibody titer was constant across fecal egg counts. We extended these into "animal models," in which we allowed the permanent environment variance in antibody titer to vary with fecal egg count and age, and tested for additive genetic variance in these slopes. We thus assessed whether individuals varied in how antibody titers changed with age or parasite egg excretion and whether these changes had a heritable component.

Associations between Antibody Titer and Annual Survival and Reproduction. We then tested whether antibody titer was associated with survival and reproductive performance of hosts, using generalized linear and linear mixed-effects models in the lme4 R package (Bates et al. 2012), as follows. We analyzed predictors of overwinter survival (a binary response variable) separately for male and female lambs $(N=292$ and 276 , respectively), yearlings $(N=$ 94 and 97, respectively), and adults ( $\geq 2$ years; $N=97$ and 239, respectively) of known year of death. We fitted a binomial error distribution and specified a logit link function in all generalized linear mixed models of survival. We included year of measurement as a random effect in all survival models and individual identity as a random effect in models of adult survival. We fitted linear and quadratic fixed effects of antibody titer, body weight, and fecal egg count plus linear and quadratic fixed effects of age in models of adults and all two-way interactions. We also tested whether a population crash (as a two-level fixed factor) predicted survival independently or via interactions with other factors. Crash was included as a general indicator of environmental quality (e.g., Hayward et al. 2011), based on the observation that crash winters are associated with high mortality and very poor condition of survivors and therefore represent extremely adverse conditions and periods of strong selection (Clutton-Brock et al. 1992).

Our analyses of reproductive performance considered the sexes separately and focused on adults only ( $\geq 2$ years) of known reproductive output. We tested predictors of annual reproduction (as a binary variable, dividing individuals into those who did versus did not reproduce) in generalized linear mixed-effects models with a binomial 
error distribution and a logit link function. Also, annual fecundity was calculated as the number of offspring each female bore and each male sired in a year $(0,1$, or 2 lambs for 401 females; 0-46 lambs for 128 males). These data were $\log (+1)$ transformed and analyzed in linear mixed models with Gaussian error. We assessed breeding in the cycles before and after each antibody measurement. We included random effects for year and individual identity and fitted both linear and quadratic fixed effects of August antibody titer, age, body weight, fecal egg count, and all two-way interactions. We also tested whether population crash (as a two-level fixed factor) predicted reproduction independently or via interactions with other factors. We assessed the significance of associations of fixed factors with survival and reproduction using Wald statistics for generalized and $F$-statistics for linear mixed-effects models.

Associations between Individual Resistance and Tolerance. Finally, we assessed relationships among individual-level antibody titers, body weight, and nematode fecal egg counts (and thus tolerance), using bivariate random regression models, as follows. Antibody titer and weight were fitted as response variables in bivariate linear mixed-effects models using ASReml 3.0. Age, sex, and standardized fecal egg count were fitted as fixed effects for both traits. We also fitted random effects of individual identity and year, as well as the residual component. We could therefore estimate the individual, annual, and residual covariance between body weight and antibody titer in this "baseline model." We also fitted the random effect of assay date to antibody titer data. Finally, we fitted an interaction between individual identity and fecal egg count for weight, thus generating a bivariate random regression model for variation in tolerance (individual-level variation in slope, similar to analyses of tolerance across the entire host-parasite time series, 1988-2012, in Hayward et al., forthcoming). This model did not successfully converge with heterogeneous residuals, but because we have found that inferences about tolerance variation were consistent whether residual variance was heterogeneous or homogeneous (Hayward et al., forthcoming), we here assumed residual variance for both traits to be constant across the range of fecal egg counts. We then modelled permanent environment and additive genetic variance in the traits as a function of fecal egg count, using bivariate animal models. We tested the significance of covariance terms by constraining each to zero in turn, and comparing the $\log \mathrm{L}$ of the constrained model with that of the full model using likelihood ratio tests as above. This allowed us to estimate, at both the individual and genetic level, covariance between resistance (estimated as T. circumcincta-specific antibody titer) and tolerance (estimated as the individuallevel association between body weight and fecal egg count) while simultaneously assessing covariance of both resistance and tolerance with body weight, as an estimate of condition.

\section{Results \\ Sheep and Parasite Dynamics}

We are motivated to understand the host-parasite dynamics over the past 25 years on St. Kilda, so we begin by presenting that time series: the number of sheep in the Village Bay study population and the August mean nematode fecal egg count of the young of each year (thus considering lambs as annual sentinels for nematode transmission) from 1988 to 2012 (fig. 2). We found that host population size and nematode egg excretion are positively correlated $\left(r^{2}=0.41 ; N=25 ; P=.042\right.$; see also Wilson et al. 2004). Below, we discuss this pattern in light of theory as well as our new data on resistance and tolerance.

\section{Associations between Antibody Titer and Parasite Burden}

We measured titers of antibody binding Trichostrongylus circumcincta adult nematode antigens in samples collected across an 11-year portion of the time series (1997-2007). We expected that if the antibodies are a useful marker of resistance, titer should be negatively associated with fecal egg count (Graham et al. 2011). Indeed, our best-supported model suggested a negative linear association between fecal egg count and titer $\left(\chi_{1}^{2}=5.0 ; P=.025\right.$; fig. 3 ; table A2), accounting for significant age- and sexdependent variation. Lambs exhibited considerably lower titers than older individuals, among whom titer remained constant with age, and females generally exhibited higher titers than males (fig. A1, available online; table A3). Nonlinear functions and interactions with age or sex did not improve model fit. The effect size for the association between titer and fecal egg count was modest: when we calculated the conditional $R^{2}$ (proportion of variance explained by fixed + random effects) and the marginal $R^{2}$ (proportion of variance explained by fixed effects; Nakagawa and Schielzeth 2013), we found that fixed and random effects together explained $61.8 \%$ of the variance, and fixed alone explained $30.0 \%$; when we dropped fixed effects of age and sex, the conditional $R^{2}$ and marginal $R^{2}$ were estimated at $45.2 \%$ and $6.3 \%$, respectively. Still, the $6.3 \%$ effect size for fecal egg count within age and sex classes, plus associations across age and sex classes, suggest that antibody titer does reflect variation in resistance. 


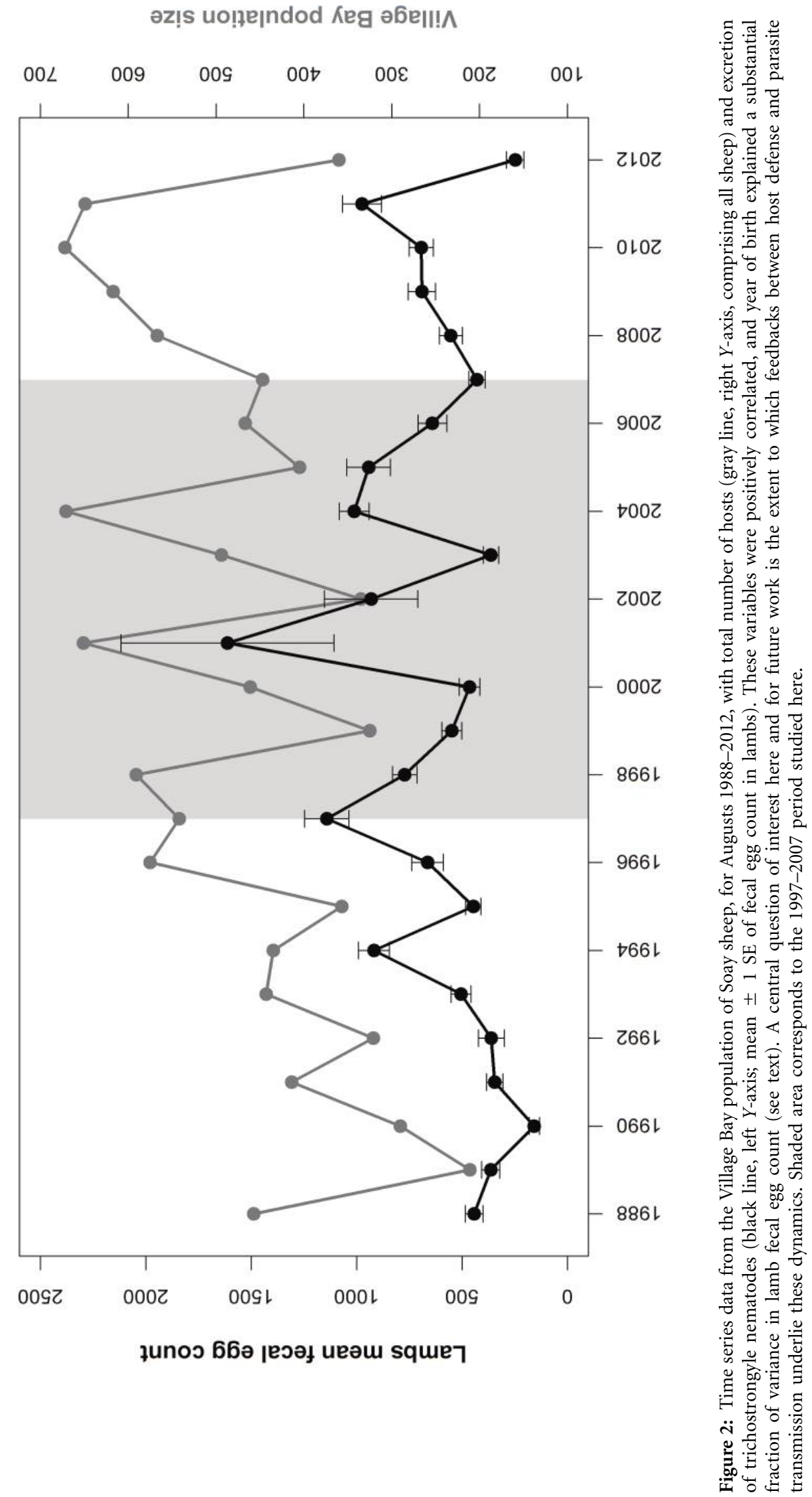

This content downloaded from 139.153.115.011 on November 16, 2017 01:50:08 AM All use subject to University of Chicago Press Terms and Conditions (http://www.journals.uchicago.edu/t-and-c). 


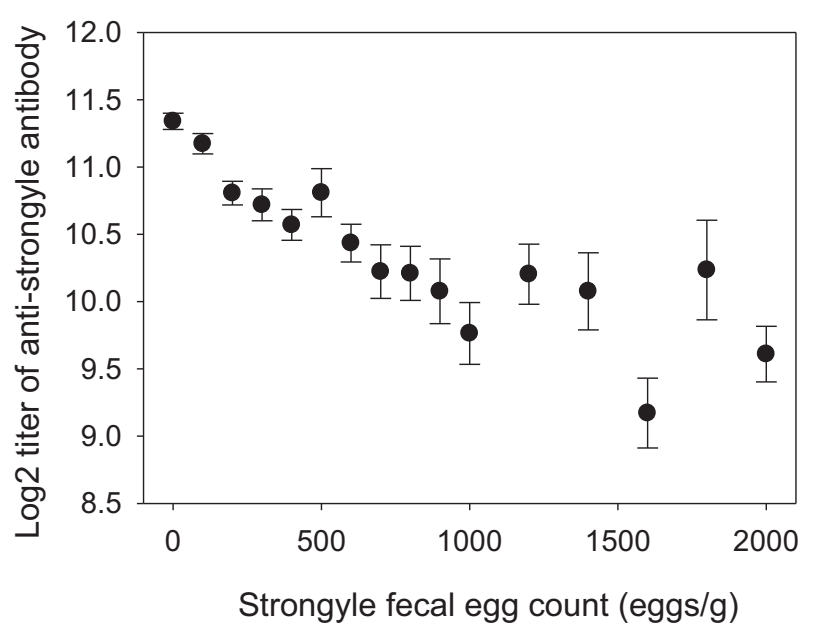

Figure 3: Strongyle fecal egg count was negatively associated with plasma anti-Teladorsagia circumcincta antibody ( $\mathrm{TcAb}$ ) titer (on a $\log 2$ scale). This negative association was significant in linear mixedeffects models accounting for host age, weight, and sex (table A2, available online). Points show mean titers ( $\pm 1 \mathrm{SE}$ ) within groupings of fecal egg count means, reflecting counts in 22 to 549 samples. Fecal egg counts are in multiples of $100 \mathrm{eggs}^{-1}$; data are binned in intervals of 100 up to 1,000 eggs $\mathrm{g}^{-1}$ and intervals of 200 thereafter (e.g., data binned at 1,200 are from sheep with 1,001-1,200 eggs $\mathrm{g}^{-1}$; data binned at 1,400 are from those with $1,201-1,400)$. Data binned at 2,000 are from sheep with $>1,900(N=57)$.

\section{Individual and Genetic Variation in Antibody Titer and Body Weight}

We next assessed individual-level variability and heritability of body weight and nematode-specific antibody titer. There was significant additive genetic variance for weight, accounting for approximately one-quarter $(0.25 \pm 0.05)$ of the total phenotypic variance and within the range of previous estimates from this population (Coltman et al. 2001; Robinson et al. 2009). In addition, there was a permanent environment effect of $0.37 \pm 0.05$ which, summed with the additive genetic effect, meant that the proportion of phenotypic variance in body weight explained by individual identity (repeatability) was approximately $62 \%$ $\left(V_{\mathrm{IND}}=\left(V_{\mathrm{A}}+V_{\mathrm{PE}}\right) / V_{\mathrm{P}}\right)$. Finally, there was an annual component of $0.07 \pm 0.03$, but the maternal effect was only $0.04 \pm 0.02$ and was not significant. There was also considerable additive genetic variance for antibody titer, with a significant heritability of $0.13 \pm 0.04$. Combined with the significant permanent environment effect of $0.20 \pm 0.04$, this gave a repeatability of around $33 \%$. There was a small effect of assay date $(0.10 \pm 0.03)$ but negligible maternal $(0.03 \pm 0.02)$ and annual $(0.01 \pm 0.01)$ effects. Table A4 shows details of the statistical significance of these variance components.

\section{Individual Variation in Associations between Antibody Titer, Parasite Burden, and Age}

We next explored individual-level variation in associations among antibody titer, fecal egg count, and age using random regression models. There was no statistical support for overall individual variance in changes in these associations (table 1; models 1-3). However, there was support for separate additive genetic and permanent environment variation in changes in titer with age (table 1; model 8). This means that there is measurable heterogeneity among individuals in antibody titers across the life span, and that not only variance in mean antibody titers but also in ageassociated trajectories of antibody production have an additive genetic basis. This could result, for instance, from genetic variation in rates of development and/or senescence in immune function.

\section{Associations between Antibody Titer and Annual Survival and Reproduction}

Despite the overall negative association with fecal egg count (fig. 3), pan-isotype nematode-specific antibodies were predictive of survival only in female lambs: independent of fecal egg count, high antibody titer was associated with survival $\left(b=0.36 \pm 0.16 \mathrm{SE} ; \chi_{1}^{2}=2.22\right.$; $P=.026)$ controlling for the detrimental effect of population crashes $\left(b=-3.09 \pm 1.48 \mathrm{SE} ; \chi_{1}^{2}=2.09 ; P=\right.$ .037 ) and independent of a marginally nonsignificant, positive effect of weight $\left(b=0.18 \pm 0.09 \mathrm{SE} ; \chi_{1}^{2}=1.95\right.$; $P=.051)$. There was no evidence of a quadratic association between titer and survival $\left(\chi_{1}^{2}=1.30, P=.22\right)$. Interestingly, the effect of titer interacted with whether survival of female lambs was measured across a crash winter (crash-by-titer estimate $=-0.90 \pm 0.37 \mathrm{SE} ; \chi_{1}^{2}=$ $2.45 ; P=.014)$. The negative interaction suggests that the weak positive association with survival is expressed during noncrash winters but that during crash winters, this positive effect is diminished. This is in accordance with the finding that survival selection on lambs due to nematodes is strongest in noncrash years (Hayward et al. 2011): because lamb survival is so low during crash years $(<10 \%$ in five such years), any effect of resistance or titer may become unimportant or undetectable. It may further suggest a survival cost of investment in immunity by female lambs during crashes. Survival of all other age and sex classes was predicted only by body weight, whether a population crash occurred, and (for adults) age, as previously described for other subsets of the data (e.g., Catchpole et al. 2000; Wilson et al. 2007; Hayward et al. 2011), with no evidence for linear or quadratic effects of titer on survival nor via interactions (all antibody-associated terms in these models: $\chi_{1}^{2}<1.39, P>.16$ ). Thus, only for female 
Table 1: A comparison of random regression models investigating changes in antibody titer with increasing strongyle fecal egg count (FEC) and age

\begin{tabular}{|c|c|c|c|c|c|c|}
\hline Model & ID treatment & $\log L$ & Versus & $\chi^{2}$ & $\mathrm{df}$ & $P$ \\
\hline \multicolumn{7}{|c|}{ Individual-level variation: } \\
\hline $\mathbf{0}$ & ID + year + date & $-1,742.25$ & & & & \\
\hline 1 & $\mathrm{ID} \times \mathrm{FEC}$ & $-1,741.13$ & 0 & 2.24 & 2 & .326 \\
\hline 2 & ID $\times$ age & $-1,740.90$ & 0 & 2.70 & 2 & .259 \\
\hline 3 & $\mathrm{ID} \times(\mathrm{FEC}+$ age $)$ & $-1,738.88$ & 2 & 4.04 & 3 & .257 \\
\hline \multicolumn{7}{|c|}{ Genetic variation: } \\
\hline 4 & $V_{\mathrm{A}}+V_{\mathrm{PE}}$ & $-1,729.71$ & 0 & 8.98 & 1 & .030 \\
\hline 5 & $V_{\mathrm{PE}} \times \mathrm{FEC}$ & $-1,728.86$ & 4 & 1.70 & 2 & .427 \\
\hline 6 & $\left(V_{\mathrm{PE}}+V_{\mathrm{A}}\right) \times \mathrm{FEC}$ & $-1,726.81$ & 5 & 4.10 & 2 & .129 \\
\hline 7 & $V_{\mathrm{PE}} \times$ age & $-1,727.91$ & 4 & 3.6 & 2 & .165 \\
\hline 8 & $\left(V_{\mathrm{PE}}+V_{\mathrm{A}}\right) \times$ age & $-1,721.11$ & 7 & 13.6 & 2 & $<.001$ \\
\hline 9 & $V_{\mathrm{PE}} \times(\mathrm{FEC}+$ age $)$ & $-1,726.12$ & 7 & 3.58 & 3 & .311 \\
\hline 10 & $V_{\mathrm{PE}} \times(\mathrm{FEC}+$ age $)+V_{\mathrm{A}} \times \mathrm{FEC}$ & $-1,724.33$ & 9 & 3.58 & 2 & .167 \\
\hline 11 & $V_{\mathrm{PE}} \times(\mathrm{FEC}+$ age $)+V_{\mathrm{A}} \times$ age & $-1,719.49$ & 9 & 13.26 & 3 & $<.001$ \\
\hline 12 & $V_{\mathrm{PE}}+V_{\mathrm{A}} \times(\mathrm{FEC}+$ age $)$ & $-1,718.66$ & 11 & 1.66 & 3 & .646 \\
\hline
\end{tabular}

Note: We fitted increasingly complex variance components structures, allowing the slope of titer on FEC and age to vary across individuals (models $1-3$ ) and then separating the individual-level variation (ID) into its additive genetic $\left(V_{\mathrm{A}}\right)$ and permanent environment $\left(V_{\mathrm{PE}}\right)$ components (models 4-12). The best-supported individual and genetic variation models are shown in bold type. $\log \mathrm{L}=\log$ likelihood.

lambs did the pan-isotype nematode-specific antibody provide any additional predictive power for overwinter survival.

We also tested for associations between T. circumcinctaspecific antibody titer and annual reproduction. We detected no significant associations with breeding in the season before antibody measurement for either sex (all titerassociated terms $\chi_{1}^{2}<0.80$ and $P>.43$ ), suggesting that breeding did not result in an up- or downregulation of immunoglobulin production. For breeding in the spring following antibody measurement, however, we found the following statistically significant associations. In adult males, higher August antibody titer was associated with a lower probability of siring offspring in the ensuing breeding season, and the association remained when we controlled for a significant effect of weight (titer $b=$ $-0.29 \pm 0.14$ SE; $\chi_{1}^{2}=2.08 ; P=.038$; fig. 4). There was no evidence for an effect of age, a quadratic effect of titer, or any interaction of titer with other terms in models of male reproduction (all $\chi_{1}^{2}<0.70 ; P>.49$ ). For females, we also detected a negative association between titer and subsequent reproduction, but the effect depended upon host weight: independent of a significant effect of host age, higher August titers of nematode-specific antibody were associated with lower probability of giving birth the following April, in females of high body weight $\left(\chi_{1}^{2}=\right.$ 1.06 and $P=.29$ for the main effect of titer; $\chi_{1}^{2}=0.39$ and $P=.70$ for the main effect of weight; and $b=$ $-0.10 \pm 0.04 \mathrm{SE} ; \chi_{1}^{2}=2.19 ; P=.029$ for the titer-weight interaction; fig. 5). No other fixed effects nor any quadratic or interactive effects of titer were significant (all $P>.15$ ). These associations were marginally nonsignificant in linear mixed-effects models of annual fecundity controlling for age and weight $\left(F_{1,125}=3.15 ; P=.078\right.$ for a negative titer-fecundity association in males; $F_{1,387}=2.36 ; P=.13$ in females). The patterns were therefore more strongly associated with whether or not an individual bred than with the number of offspring.

\section{Associations between Individual Resistance and Tolerance}

Finally, we assessed whether variation in resistance was associated with variation in tolerance via an oft-assumed trade-off between the two modes of defense (Restif and Koella 2003; Miller et al. 2006; Best et al. 2008). We used bivariate random regression to quantify the relationship between individual-level resistance (estimated as the intercept of antibody titer) and tolerance (estimated as the individual slope of weight on fecal egg count). In case the relationship was confounded by variation in individual condition (Kruuk et al. 2008), we assessed the covariance of resistance and tolerance with body weight, and tested for resistance-tolerance covariance once associations with weight were taken into account. Full results summarized below appear in table A5.

We began with a baseline bivariate model of antibody titer and weight that included fixed effects of age, sex, and fecal egg count as well as random effects of individual identity and year of measurement. We found weak but significant positive residual covariance $\left(\rho_{\mathrm{R}}=+0.077 \pm\right.$ 


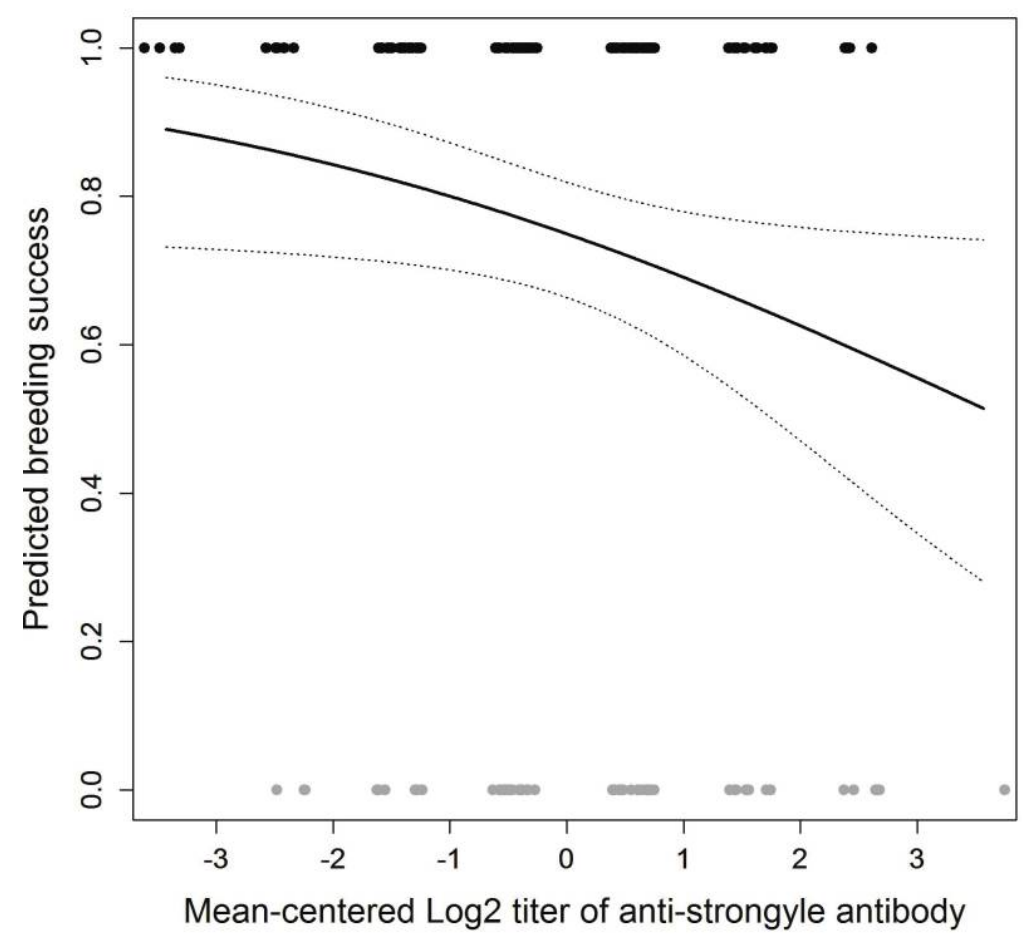

Figure 4: In adult males (aged $\geq 2 \mathrm{yr}$ ), high Teladorsagia circumcincta-specific antibody titer measured in August was associated with reduced subsequent probability of siring offspring. Gray dots represent jittered titers of sheep that did not breed; black dots represent jittered titers of sheep that did breed. Lines represent significant estimated effects $(+95 \%$ confidence interval) from generalized linear mixed-effects models.

0.032 (estimate $\pm \mathrm{SE}$ ); $\chi_{1}^{2}=5.58 ; P=.018$ ) and marginally nonsignificant positive individual covariance $\left(\rho_{\text {IND }}=+0.095 \pm 0.049 ; \chi_{1}^{2}=3.64 ; P=.056\right)$ between antibody titer and weight. We next added the random interaction for individual identity with fecal egg count, to model individual variance in tolerance (in keeping with our analysis of tolerance variation across the whole St. Kilda study period; Hayward et al., forthcoming). The individual-level correlations estimated by this model are shown in figure 6 . Inclusion of individual tolerance variation did not alter the marginally nonsignificant, weakly positive individual covariance between weight and antibody titer $\left(\rho_{\mathrm{IND}}=+0.090 \pm 0.049 ; \chi_{1}^{2}=3.20 ; P=\right.$ $.074)$. There was no evidence of individual-level covariance between weight and tolerance $\left(\rho_{\mathrm{IND}}=-0.012 \pm 0.148\right.$; $\chi_{1}^{2}=0.02 ; P=.888$ ), in accord with our finding that tolerance (i.e., the slope) is independent of body weight (i.e., the intercept) across the entire study period (Hayward et al., forthcoming). Importantly, even with titer-weight covariance accounted for, tolerance did not covary significantly with antibody titer of individuals $\left(\rho_{\mathrm{IND}}=\right.$
$+0.176 \pm 0.189 ; \chi_{1}^{2}=0.96 ; P=.327 ;$ fig. 6$)$. We therefore found no evidence of a trade-off between resistance and tolerance.

We further investigated the association between body weight and antibody titer, given that we had found individual-level heterogeneity in how titer varied with age (table 1). When, instead of adding terms for tolerance variation to the baseline bivariate model, we added the interaction for individual identity with age (across which we expected that both weight and titer were changing), we found significant individual variance in age-associated changes in weight $\left(\chi_{3}^{2}=61.50 ; P<.001\right)$. This inclusion strengthened the positive individual covariance between weight and antibody titer $\left(\rho_{\text {IND }}=+0.102 \pm 0.050 ; \chi_{1}^{2}=\right.$ $3.98 ; P=.046)$ and revealed significant positive covariance between age-associated changes in weight and weight itself $\left(\rho_{\mathrm{IND}}=+0.208 \pm 0.087 ; \chi_{1}^{2}=5.82 ; P=.016\right)$ but no significant covariance of age-associated changes in weight with antibody titer $\left(\rho_{\text {IND }}=-0.018 \pm 0.106 ; \chi_{1}^{2}=0.04\right.$; $P=.841$ ). Thus the association of weight with titer did not change across life span. Finally, we extended the model 

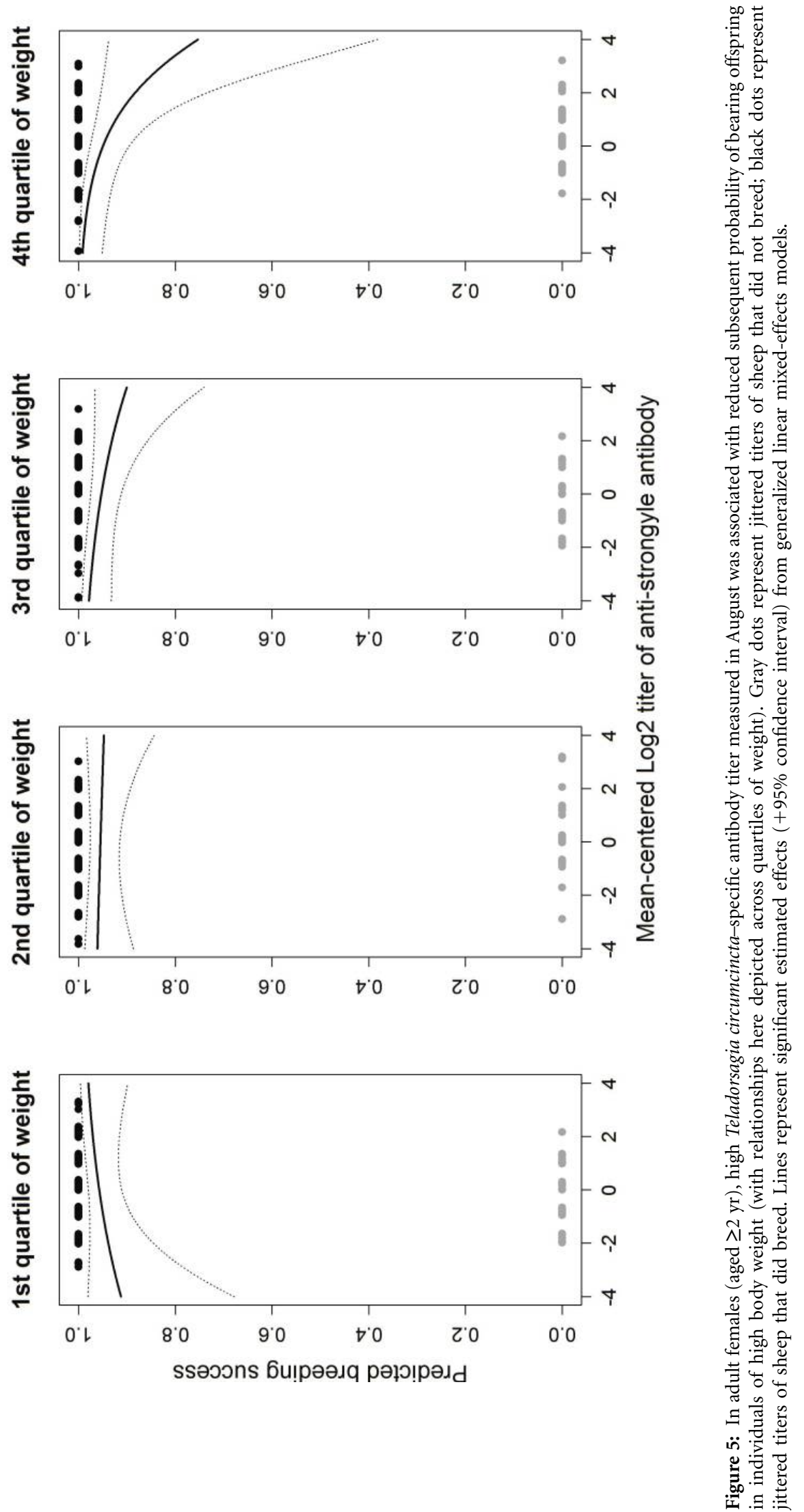

This content downloaded from 139.153.115.011 on November 16, 2017 01:50:08 AM All use subject to University of Chicago Press Terms and Conditions (http://www.journals.uchicago.edu/t-and-c). 


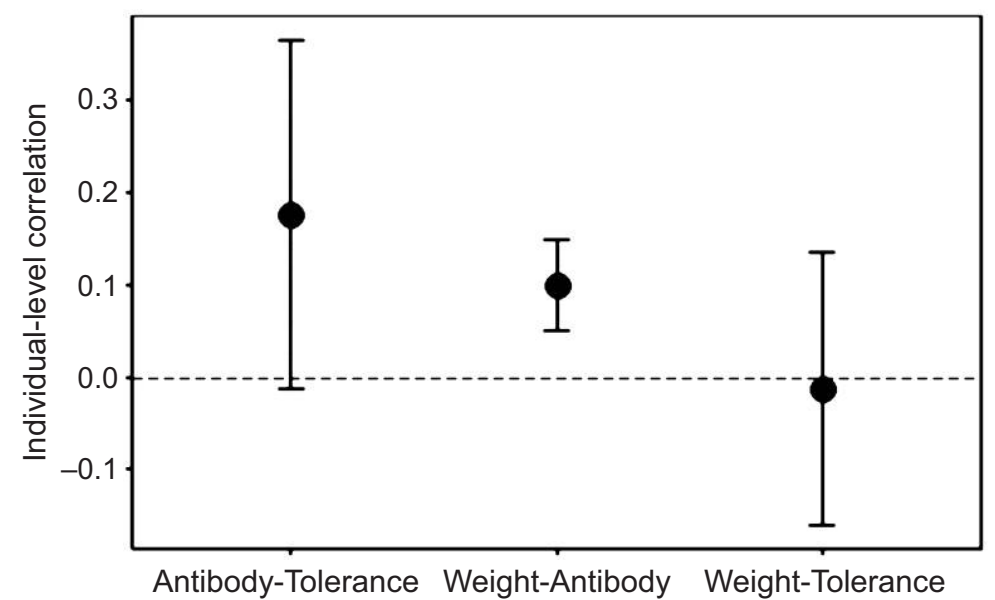

Figure 6: Individual-level correlations from a bivariate model of body weight and antibody titer, including a random slope of body weight on strongyle fecal egg count to quantify individual variation in tolerance. Points show estimated correlations \pm 1 SE. Significance of components is described in the text with details in table A5, available online.

to divide individual variance into additive genetic and permanent environment components. This revealed that the positive covariance between weight and antibody titer was underpinned by a significant permanent environment component $\left(\rho_{\mathrm{PE}}=+0.197 \pm 0.096 ; \chi_{1}^{2}=4.20 ; P=\right.$ $.040)$ but not a significant additive genetic component $\left(\rho_{\mathrm{A}}=-0.066 \pm 0.159 ; \chi_{1}^{2}=0.18 ; P=.671\right)$.

\section{Discussion}

Theory predicts profoundly different epidemiological and genetic ramifications of resistant versus tolerant individual defense strategies in host populations (Best et al. 2008; Boots et al. 2009). Although others have undertaken empirical tests of the theory's assumptions (e.g., resistancetolerance trade-offs at the individual level; Fineblum and Rausher 1995; Råberg et al. 2007; Rohr et al. 2010) and predictions (e.g., genetic variation in resistance but not tolerance; Blanchet et al. 2010; Lefevre et al. 2011), none have yet tackled simultaneous operation of resistance and tolerance across biological scales (molecules to populations) in a natural population. We are laying the groundwork for such tests in a field system in which longitudinally monitored individual hosts (Clutton-Brock and Pemberton 2004) experience strong parasite-mediated selection (Gulland 1992; Craig et al. 2006; Hayward et al. 2011) in a closed, island population in which feedbacks might be observable. Here, we present evidence that across 11 years of the $>25$-year time series, parasite-specific antibody titer, a proposed marker of resistance against trichostrongyle nematodes (i) was negatively associated with nematode fecal egg counts; (ii) exhibited substantial individual and genetic variation, both in mean antibody titer and how it changed with host age; (iii) was negatively associated with annual breeding success but was not generally associated with host survival; and (iv) did not exhibit a detectable trade-off with individual tolerance. These findings strengthen the empirical basis for testing theoretical predictions about reciprocal selection in this host-parasite system.

We expect that the type, variability, and strength of parasite-mediated selection on hosts on the one hand, and immune selection on parasites on the other, will combine with epidemiological feedbacks and environmental conditions to generate the observed time series. Although such deep understanding of system behavior will remain a longer-term aim, time series data of annual host population size in relation to mean lamb fecal egg count (a measure of transmission intensity correlated with springsummer density of infective nematode larvae on the pasture; Wilson et al. 2004) did reveal a significant positive correlation. This pattern accords with a comparative study of density-dependent transmission of strongyle nematodes across mammals (Arneberg et al. 1998). However, accounting for intra-annual variation (e.g., Coltman et al. 2001; Beraldi et al. 2007), interannual variation explains significant variance in the burden of nematodes harbored by individual Soay lambs independent of host density (Gulland and Fox 1992; Wilson et al. 2004; Hayward et al. 2009, 2010). Indeed, evolutionary epidemiological theories of host defense (e.g., Boots et al. 2009) posit frequency-dependent as well as density-dependent effects on transmission: heterogeneous host defense strategies are also expected to shape the dynamics of parasite abundance. For example, if adult sheep that survive a population crash are relatively nematode-resistant and well fed (due to re- 
duced competition for forage; Crawley et al. 2004), then the relative postcrash defensive composition of the host population should contribute to a sustained reduction in parasite transmission. However, any costs of resistance would be expected eventually to reduce the frequency of resistance in the population (Boots et al. 2009), such that first more susceptible hosts and then parasite abundance should rebound. We are only beginning to measure the processes that could contribute to these dynamics, but here, we strengthen the empirical basis for testing such models with a putative phenotypic marker of nematode resistance.

Previous studies in domesticated livestock have shown immune phenotype may exhibit considerable additive genetic variance (Strain et al. 2002; Gutierrez-Gil et al. 2010), but estimates in field studies of wild vertebrates are rare (though see Pitala et al. 2007; Bonneaud et al. 2009; Kim et al. 2013). We discovered a significant additive genetic component to nematode-specific antibody titer, with a repeatability of 0.31 and an estimated heritability of 0.13 . This is lower than reported previously in analyses splitting the population into age groups (Brown et al. 2013) but similar to heterogeneity and heritability of self-reactive antibodies in the same sample set (repeatability of $0.37 \pm$ 0.04 and heritability of $0.13 \pm 0.03$; Graham et al. 2010). We further observed that changes in titer across the life span-potentially in rates of acquisition and decay of resistance-are under additive genetic control.

A key question is how genetically controlled variation in nematode-specific antibody titers is maintained in the Soay sheep. One major evolutionary explanation centers on immune-mediated life-history trade-offs, whereby investment in immunity may promote survival but detract from investment in reproduction (Sheldon and Verhulst 1996; Demas and Nelson 2012). We previously found that self-reactive antibodies were associated with enhanced annual survival probability among ewes but reduced annual reproduction in both rams and ewes (Graham et al. 2010). We therefore analyzed associations between parasite-specific antibodies and components of fitness. Extensive previous work on the Soay sheep has focused primarily on fecal egg count as an inverse measure of resistance against strongyle nematodes (e.g., Gulland 1992; Smith et al. 1999; Wilson et al. 2004; Craig et al. 2006; Hayward et al. 2011). Such a metric has enabled estimation of the costs of parasitism and benefits of resistance for host survival (Gulland 1992; Craig et al. 2006; Hayward et al. 2011) and fecundity (Hayward et al. 2011) but has not yielded evidence of any costs of resistance. Parasite density data can, in principle, be used for such tests. For example, a parabolic association between protozoan parasite burden and overwinter survival in a wild blue tit population (with low host survival rates at both low and high parasite burdens) suggested costs of both resistance and parasitism in that system (Stjernman et al. 2008). However, similar analysis testing for nonlinear selection on fecal egg counts in the Soay sheep revealed no such pattern (Hayward et al. 2011).

Here, nematode-specific antibody titers did reveal modest negative associations with subsequent reproductive success. In adult males, the association was via a main effect. However, in adult females, the association was only evident in individuals of high body weight, suggesting a three-way complexity to the underlying physiological trade-offs (as opposed to a simpler two-way trade-off between immunity and fecundity). For example, in ewes, perhaps resources such as protein must be allocated among antibodies, offspring, and maintenance of weight. Some sacrifice body weight to invest in antibodies and lambs; others sacrifice lambs to invest in antibodies and weight. This might be construed as a trade-off between current and future reproduction and suggests that different trade-offs may pertain for males and females, perhaps associated with asymmetries in the type and timing of investments in reproduction. In both sexes, the associations were independent of other major predictors of reproductive success (e.g., weight in males [Preston et al. 2003] and age in females [Hayward et al. 2013]). Whether this apparent cost of nematode resistance is due to hormonal immunosuppression of resistance (Norris and Evans 2000) and/or resource allocation among life-history imperatives (Sheldon and Verhulst 1996; Demas and Nelson 2012) or fighting coinfections (Craig et al. 2008) is an area for future investigation. The evidence for heritable heterogeneity in a costly resistance trait suggests, however, that many of the preconditions for negative frequency-dependent selection on resistance (Boots et al. 2009) are met.

Of course, the evolutionary epidemiological predictions hinge on there being benefits as well as costs of resistance. We found a significant negative association between antibody titer and fecal egg count, both among and within age and sex classes, as would be predicted if the antibody marker (or a correlated immune effector mechanism) afforded resistance (Bradley and Jackson 2008; Graham et al. 2011). However, the effect was relatively weak, and we found an association with survival only among female lambs over winters when the population did not crash (consistent with analyses of nematode-associated survival selection on lambs; Hayward et al. 2011). Thus, for the titers of all antibody isotypes binding antigens of adult Trichostrongylus circumcincta reported here, we found no compelling general association with survival and thus no individual-level benefit of resistance. In the companion study in which we separately measured distinct antibody isotypes, we report that adult females with the highest titers of isotype IgG binding larval antigens did not have lower fecal egg counts but were nonetheless significantly more 
likely to survive a population crash (Nussey et al. 2014). The antigenic target and functional type of antibody thus appear crucial. Indeed, different isotypes confer differential protection against strongyle nematodes (Stear et al. 1995, 1997; Strain et al. 2002; Williams et al. 2010; Williams 2012), so future studies of Soay sheep survival across the whole time series might usefully focus on IgG specific to larval antigens or to defined protein antigens with protective capacity (Nisbet et al. 2013) rather than a conglomerate titer of all isotypes binding antigenically complex adult antigen extracts. Conversely, isotypes such as IgA or IgE directed against antigens of adult nematodes or their eggs may prove the best predictors of fecal egg excretion rates (Stear et al. 1995, 1997; Strain et al. 2002; Williams et al. 2010; Williams 2012). These considerations will inform the next steps in our empirical work. For now, we conclude that our pan-isotype anti-T. circumcincta antibody metric, though tractable across a large sample set, captures transmission-reducing effects of resistance but masks survival-promoting effects.

Why, then, did we detect negative associations of titer with reproduction? Any antibody molecule, survival-promoting or not, potentially costs protein energy (e.g., Martin et al. 2007) that might otherwise be invested in reproduction. Similarly, reproductive hormones may suppress antibody titers regardless of specificity (Norris and Evans 2000). Indeed, the mechanics of B cell and plasma cell development are likely to generate many antibodies of the wrong isotype and specificity to provide any resistance at all. Thus, high antibody titers that modestly suppress fecal egg counts but have no effect on survival may nonetheless generate a reproductive cost to adults with such immune investment. Further empirical work is required to assess this hypothesis and to ascertain whether the IgG that may promote survival is associated, on its own, with reduced reproduction. It may be that, at least for adults in this population (see Hayward et al. 2011), the benefits of IgG-associated nematode resistance are expressed during population crashes (Nussey et al. 2014), while the costs of resistance are expressed in reduced breeding rates between crashes. Mathematical and demographic modeling will be required explicitly to assess dependence of any associations between resistance and reproduction or survival upon the phase of the population dynamics or condition of the individual and to understand how frequency-dependent selection on host defense may operate in the context of density-dependent processes (e.g., Mappes et al. 2008) that also shape host-parasite dynamics on the island (Coulson et al. 2001; Wilson et al. 2004).

Epidemiological feedbacks are expected to generate genetic heterogeneity in resistance and fixation of tolerance in host populations, unless tolerance variation is maintained by a trade-off against resistance or intrinsic costs of tolerance (Simms 2000; Miller et al. 2005, 2006; Best et al. 2008; Boots et al. 2009). For example, genetic heterogeneity in resistance that trades off with tolerance (e.g., Råberg et al. 2007) could maintain tolerance heterogeneity. Taken together, our results accord with many of these expectations. Our animal model analysis of antibody titers here joins other evidence (based on fecal egg counts) of heterogeneous and heritable nematode resistance among the Soay sheep (Gulland et al. 1993; Paterson et al. 1998; Smith et al. 1999; Coltman et al. 2001; Beraldi et al. 2007). Furthermore, here and in analyses of the entire parasitological time series (Hayward et al., forthcoming), we found significant individual but not additive genetic variation in nematode tolerance. However, our results provide no evidence of a resistance-tolerance trade-off. Indeed, in this system, resistance and tolerance appear not to be mutually exclusive alternatives but instead operate simultaneously or sequentially within individuals (e.g., Behnke et al. 1992; Restif and Koella 2004; Carval and Ferriere 2010). Interestingly, mixed strategies are predicted to evolve when resistance and tolerance are not both costly to reproduction (Restif and Koella 2004). We have found that nematode tolerance is positively associated with lifetime breeding success of the Soay sheep (Hayward et al., forthcoming). Therefore, perhaps the key to host-parasite dynamics in the Soay population instead lies in how resistance and tolerance each relates independently to fitness (Restif and Koella 2004).

We note that our resistance and tolerance estimates were generated with error and may be only weakly correlated with true determinants of resistance and tolerance. It would be of interest to correct such deficiencies and to test for trade-offs using an instantaneous tolerance estimate (such as a physiological marker of tolerance, of which there are several candidates in livestock; Glass 2012) generated at the individual level at multiple time points. Such estimates might enable analysis of whether tolerance varies across age, condition, period of the reproductive cycle, or environmental conditions, or indeed whether instantaneous (as opposed to lifelong) tolerance covaries with resistance. Ideally, we would thus obtain physiological data on host nutrition, condition, and tolerance to accompany antibody data of diverse specificities and functional types. This more comprehensive data set could promote our ability to achieve linkages between defense molecules and their selective and population dynamical consequences. These remain challenges for future work.

\section{Outlook}

The 25-year host-parasite time series on St. Kilda is arguably consistent with negative frequency-dependence, for example, if the benefits of resistance promote survival of 
population crashes (Nussey et al. 2014); but then after a crash, parasite prevalence drops so low that costs of resistance outweigh benefits and relatively susceptible sheep outbreed resistant ones. Such a mechanism could plausibly combine with density dependence to generate the observed patterns of host and parasite abundance and maintain genetic heterogeneity for host resistance. However, much demographic and mathematical modeling as well as empirical work remains to be done. Indeed, close integration of individual and population scale data with models is warranted. Testing evolutionary and ecological theory across scales of biology may be ambitious, but it is essential to advance our understanding of eco-evolutionary feedbacks and host-parasite coevolution in nature.

\section{Acknowledgments}

This work was supported by the United Kingdom Natural Environment and Biotechnology and Biological Sciences Research Councils (including BBSRC Fellowships to D.H.N. and A.L.G.); the Wellcome Trust; the Research and Policy for Infectious Disease Dynamics (RAPIDD) program of the Science and Technology Directorate, US Department of Homeland Security; and the Fogarty International Center of the National Institutes of Health. A.D.H. is funded by a European Research Council (ERC) grant to V. Lummaa. We thank the National Trust for Scotland and Scottish Natural Heritage for permission to work on St. Kilda and the Royal Artillery Range (Hebrides) and QinetiQ for logistical support. We thank T. CluttonBrock for the long-term data set, many field volunteers for the samples, T. McNeilly for immunological advice, A. Nisbet for antigens, J. Houdijk for negative control plasma from domesticated sheep, M. Morrissey for the population pedigree, K. M. A. Morriss for laboratory assistance, and K. Wilson, A. Thomas Tate, and three anonymous reviewers for helpful comments on the draft manuscript. Finally, we thank C. Lively and the American Society of Naturalists for the opportunity to participate in this symposium.

\section{Literature Cited}

Adelman, J. S., L. Kirkpatrick, J. L. Grodio, and D. M. Hawley. 2013. House finch populations differ in early inflammatory signaling and pathogen tolerance at the peak of Mycoplasma gallisepticum infection. American Naturalist 181:674-689.

Allen, J. E., and T. A. Wynn. 2011. Evolution of Th2 immunity: a rapid repair response to tissue destructive pathogens. PLoS Pathogens 7:e1002003.

Arneberg, P., A. Skorping, B. Grenfell, and A. F. Read. 1998. Host densities as determinants of abundance in parasite communities.
Proceedings of the Roval Society B: Biological Sciences 265:12831289.

Ayres, J. S., and D. S. Schneider. 2012. Tolerance of infections. Annual Review of Immunology 30:271-294.

Barclay, V. C., D. Kennedy, V. C. Weaver, D. Sim, J. O. Lloyd-Smith, and A. F. Read. 2014. The effect of immunodeficiency on the evolution of virulence: an experimental test with the rodent malaria Plasmodium chabaudi. American Naturalist 184(suppl.):S47S57.

Barton, N. H., and M. Turelli. 1989. Evolutionary quantitative genetics: how little do we know? Annual Review of Genetics 23:337370.

Bates, D., M. Maechler, and B. Bolker. 2012. Lme4: linear mixedeffects models using S4 classes. CRAN.R-project. http://lme4.rforge.r-project.org/.

Baucom, R. B., and J. C. de Roode. 2011. Ecological immunology and tolerance in plants and animals. Functional Ecology 25:1828.

Behnke, J. M., C. J. Barnard, and D. Wakelin. 1992. Understanding chronic nematode infections: Evolutionary considerations, current hypotheses and the way forward. International Journal for Parasitology 22:861-907.

Beisel, W. R. 1977. Magnitude of the host nutritional responses to infection. American Journal of Clinical Nutrition 30:1236-1247.

Beraldi, D., A. F. McRae, J. Gratten, J. G. Pilkington, J. Slate, P. M. Visscher, J. M. Pemberton. 2007. Quantitative trait loci (QTL) mapping of resistance to strongyles and coccidia in the free-living Soay sheep (Ovis aries). International Journal for Parasitology 37: 121-129.

Best, A., A. White, and M. Boots. 2008. Maintenance of host variation in tolerance to pathogens and parasites. Proceedings of the National Academy of Sciences of the USA 105:20786-20791.

. 2009. The implications of coevolutionary dynamics to hostparasite interactions. American Naturalist 173:779-791.

Bisset, S. A. and C. A. Morris. 1996. Feasibility and implications of breeding sheep for resilience to nematode challenge. International Journal for Parasitology 26:857-868.

Blanchet, S., O. Rey, and G. Loot. 2010. Evidence for host variation in parasite tolerance in a wild fish population. Evolutionary Ecology 24:1129-1139.

Bonneaud, C., J. S. Sinsheimer, M. Richard, O. Chastel, and G. Sorci. 2009. Mhc polymorphisms fail to explain the heritability of phytohaemagglutinin-induced skin swelling in a wild passerine. Biology Letters 5:784-787.

Boots, M., A. Best, M. R. Miller and A. White. 2009. The role of ecological feedbacks in the evolution of host defence: what does theory tell us? Philosophical Transactions of the Roval Society B: Biological Sciences 364:27-36.

Bradley, J. E. and J. A. Jackson. 2008. Measuring immune system variation to help understand host-pathogen community dynamics. Parasitology 135:807-823.

Brockhurst, M. A., and B. Koskella. 2013. Experimental coevolution of species interactions. Trends in Ecology and Evolution 28:367375.

Brown, E. A., J. G. Pilkington, D. H. Nussey, K. A. Watt, A. D. Hayward, R. Tucker, A. L. Graham, et al. 2013. Detecting genes for variation in parasite burden and immunological traits in a wild population: testing the candidate gene approach. Molecular Ecology 22:757-773.

Burnham, K. P., and D. R. Anderson. 2002. Model selection and 
multimodel inference: a practical information-theoretic approach. Springer Science + Business, New York.

Carval, D., and R. Ferriere. 2010. A unified model for the coevolution of resistance, tolerance, and virulence. Evolution 64:2988-3009.

Catchpole, E. A., B. J. T. Morgan, T. N. Coulson, S. N. Freeman, and S. D. Albon. 2000. Factors influencing Soay sheep survival. Journal of the Royal Statistical Society C: Applied Statistics 49:453-472.

Charmantier, A., and D. Garant. 2005. Environmental quality and evolutionary potential: lessons from wild populations. Proceedings of the Roval Society B: Biological Sciences 272:1415-1425.

Clutton-Brock, T. H., and J. M. Pemberton, eds. 2004. Soay sheep: dynamics and selection in an island population. Cambridge University Press, Cambridge.

Clutton-Brock, T. H., O. F. Price, S. D. Albon, and P. A. Jewell. 1992. Early development and population fluctuations in Soay sheep. Journal of Animal Ecology 61:381-396.

Clutton-Brock, T. H., I. R. Stevenson, P. Marrow, A. D. MacColl, A. I. Houston, and J. M. McNamara. 1996. Population fluctuations, reproductive costs and life-history tactics in female Soay sheep. Journal of Animal Ecology 65:675-689.

Coltman, D. W., J. Pilkington, L. E. B. Kruuk, K. Wilson, and J. M. Pemberton. 2001. Positive genetic correlation between parasite resistance and body size in a free-living ungulate population. Evolution 55:2116-2125.

Coltman, D. W., K. Wilson, J. G. Pilkington, M. J. Stear, and J. M. Pemberton. 2001. A microsatellite polymorphism in the gamma interferon gene is associated with resistance to gastrointestinal nematodes in a naturally-parasitized population of Soay sheep. Parasitology 122:571-582.

Coulson, T., E. A. Catchpole, S. D. Albon, B. J. T. Morgan, J. M. Pemberton, T. H. Clutton-Brock, M. J. Crawley, and B. T. Grenfell. 2001. Age, sex, density, winter weather, and population crashes in Soay sheep. Science 292:1528-1531.

Craig, B. H., J. G. Pilkington, and J. M. Pemberton. 2006. Gastrointestinal nematode species burdens and host mortality in a feral sheep population. Parasitology 133:485-496.

Craig, B. H., L. J. Tempest, J. G. Pilkington, and J. M. Pemberton. 2008. Metazoan-protozoan parasite co-infections and host body weight in St. Kilda Soay sheep. Parasitology 135:433-441.

Crawley, M. J., S. D. Albon, D. R. Bazely, J. M. Milner, J. G. Pilkington, A. L. Tuke, T. H. Clutton-Brock, and J. M. Pemberton. 2004. Vegetation and sheep population dynamics. Pages 89-112 in T. H. Clutton-Brock and J. M. Pemberton, eds. Soay sheep: dynamics and selection in an island population. Cambridge University Press, Cambridge.

Day, T., S. Alizon, and N. Mideo. 2011. Bridging scales in the evolution of infectious disease life histories: theory. Evolution 65: 3448-3461.

Debarre, F., S. Lion, M. Van Baalen, and S. Gandon. 2012. Evolution of host life-history traits in a spatially structured host-parasite system. American Naturalist 179:52-63.

Demas, G. E. and R. J. Nelson, eds. 2012. Ecoimmunology. Oxford University Press, Oxford.

Doeschl-Wilson, A. B., S. C. Bishop, I. Kyriazakis, and B. Villanueva. 2012. Novel methods for quantifying individual host response to infectious pathogens for genetic analyses. Frontiers in Livestock Genomics 3:266.

Ferreira, A., I. Marguti, I. Bechman, V. Jeney, Â. Chora, N. R. Palha, S. Rebelo, A. Henri, Y. Beuzard, and M. P. Soares. 2011. Sickle hemoglobin confers tolerance to Plasmodium infection. Cell 145(3): 398-409.

Fineblum, W. L. and M. D. Rausher. 1995. Tradeoff between resistance and tolerance to herbivore damage in a morning glory. ture 377:517-520.

Frank, S. A. 1996. Models of parasite virulence. Quarterly Review of Biology 71:37-78.

Gilmour, A. R., B. J. Gogel, B. R. Cullis, and R. Thompson. 2009. ASReml user guide, release 3.0. New South Wales Department of Industry and Investment, Orange, Australia.

Glass, E. J. 2012. The molecular pathways underlying host resistance and tolerance to pathogens. Frontiers in Genetics 3:263.

Graham, A. L., J. E. Allen, and A. F. Read. 2005. Evolutionary causes and consequences of immunopathology. Annual Review of Ecology, Evolution, and Systematics 36:373-397.

Graham, A. L., A. D. Hayward, K. A. Watt, and J. G. Pilkington. 2010. Fitness correlates of heritable variation in antibody responsiveness in a wild mammal. Science 330:662-665.

Graham, A. L., D. M. Shuker, L. C. Pollitt, S. K. J. R. Auld, A. J. Wilson, and T. J. Little. 2011. Fitness consequences of immune responses: strengthening the empirical framework for ecoimmunology. Functional Ecology 25:5-17.

Gruner, L., J. Bouix, and J. C. Brunel. 2004. High genetic correlation between resistance to Haemonchus contortus and to Trichostrongylus colubriformis in INRA 401 sheep. Veterinary Parasitology 119: $51-58$.

Gulland, F. M. D. 1992. The role of nematode parasites in Soay sheep (Ovis aries L.). Mortality during a population crash. Parasitology 105:493-503.

Gulland, F. M. D., S. D. Albon, J. M. Pemberton, P. R. Moorcroft, and T. H. Clutton-Brock. 1993. Parasite-associated polymorphism in a cyclic ungulate population. Proceedings of the Roval Society B: Biological Sciences 254:7-13.

Gulland, F. M. D. and M. Fox. 1992. Epidemiology of nematode infections of Soay sheep (Ovis aries L.) on St-Kilda. Parasitology 105:481-492.

Gutierrez-Gil, B., J. Perez, L. F. de la Fuente, A. Meana, M. MartínezValladares, F. San Primitivo, F. A. Rojo-Vázquez, and J. J. Arranz. 2010. Genetic parameters for resistance to trichostrongylid infection in dairy sheep. Animal 4:505-512.

Hadfield, J. D., D. S. Richardson, and T. Burke. 2006. Towards unbiased parentage assignment: combining genetic, behavioural and spatial data in a Bayesian framework. Molecular Ecology 15:37153730.

Hayward, A. D., D. H. Nussey, A. J. Wilson, C. Berenos, J. G. Pilkington, K. A. Watt, J. M. Pemberton, and A. L. Graham. Forthcoming. Natural selection on individual variation in tolerance of gastrointestinal nematode infection. PLoS Biology.

Hayward, A. D., J. G. Pilkington, J. M. Pemberton, and L. E. B. Kruuk. 2010. Maternal effects and early-life performance are associated with parasite resistance across life in free-living Soay sheep. Parasitology 137:1261-1273.

Hayward, A. D., A. J. Wilson, J. G. Pilkington, T. H. Clutton-Brock, J. M. Pemberton, and L. E. B. Kruuk. 2011. Natural selection on a measure of parasite resistance varies across ages and environmental conditions in a wild mammal. $\underline{\text { Journal of Evolutionary }}$ Biology 24:1664-1676.

2013. Reproductive senescence in female Soay sheep: variation across traits and contributions of individual ageing and selective disappearance. Functional Ecology 27:184-195. 
Hayward, A. D., A. J. Wilson, J. G. Pilkington, J. M. Pemberton, and L. E. B. Kruuk. 2009. Ageing in a variable habitat: environmental stress affects senescence in parasite resistance in St Kilda Soay sheep. Proceedings of the Roval Society B: Biological Sciences 276: 3477-3485.

Henderson, C. R. 1950. Estimation of genetic parameters. Annals of Mathematical Statistics 21:309-310.

Hutchings, M. R., J. M. Milner, I. J. Gordon, I. Kyriazakis, and F. Jackson. 2002. Grazing decisions of Soay sheep, Ovis aries, on St Kilda: a consequence of parasite distribution? Oikos 96:235-244.

Jackson, F., D. Bartley, Y. Bartley, and F. Kenyon. 2009. Worm control in sheep in the future. Small Ruminant Research 86:40-45.

Jamieson, A. M., L. Pasman, S. Yu, P. Gamradt, R. J. Homer, T. Decker, and R. Medzhitov. 2013. Role of tissue protection in lethal respiratory viral-bacterial coinfection. Science 340:1230-1234.

Jones, O. R., M. J. Crawley, J. G. Pilkington, and J. M. Pemberton. 2005. Predictors of early survival in Soay sheep: cohort-, maternaland individual-level variation. Proceedings of the Roval Society B: Biological Sciences 272:2619-2625.

Kause, A. 2011. Genetic analysis of tolerance to infections using random regressions: a simulation study. Genetics Research 93:291302.

Kause, A., S. van Dalen, and H. Bovenhuis. 2012. Genetics of ascites resistance and tolerance in chicken: a random regression approach. Genes, Genomes, Genetics 2:527-535.

Kim, S. Y., J. A. Fargallo, P. Vergara, and J. Martínez-Padilla. 2013. Multivariate heredity of melanin-based coloration, body mass and immunity. Heredity 111:139-146.

Klasing, K. C. 2004. The costs of immunity. Acta Zoologica Sinica 50:961-969.

Koskella, B. 2014. Bacteria-phage interactions across time and space: merging local adaptation and time shift experiments to understand phage evolution. American Naturalist 184(suppl.):S9-S21.

Kruuk, L. E. B. 2004. Estimating genetic parameters in natural populations using the "animal model." Philosophical Transactions of the Royal Society B: Biological Sciences 359:873-890.

Kruuk, L. E. B., J. Slate, and A. J. Wilson. 2008. New answers for old questions: the evolutionary quantitative genetics of wild animal populations. Annual Review of Ecology, Evolution, and Systematics 39:525-548.

Lefevre, T., A. J. Williams, and J. C. de Roode. 2011. Genetic variation in resistance, but not tolerance, to a protozoan parasite in the monarch butterfly. Proceedings of the Roval Society B: Biological Sciences 278:751-759.

MAFF (Ministry of Agriculture, Fish and Food). 1986. Manual of veterinary parasitological laboratory techniques. Her Majesty's Stationery Office, London.

Mappes, T., M. Koivula, E. Koskela, T. A. Oksanen, T. Savolainen, and B. Sinervo. 2008. Frequency and density-dependent selection on life-history strategies: a field experiment. PLoS ONE 3:e1687.

Martin, J. G. A., D. H. Nussey, A. J. Wilson, and D. Reale. 2011. Measuring individual differences in reaction norms in field and experimental studies: a power analysis of random regression models. Methods in Ecology and Evolution 2:362-374.

Martin, L. B., 2nd, K. J. Navara, Z. M. Weil, and R. J. Nelson. 2007. Immunological memory is compromised by food restriction in deer mice Peromyscus maniculatus. American Journal of Physiology 292:R316-R320.

Medzhitov, R., D. S. Schneider, and M. P. Soares. 2012. Disease tolerance as a defense strategy. Science 335:936-941.
Mideo, N., W. A. Nelson, S. E. Reece, A. S. Ball, A. F. Read, and T. Day. 2011. Bridging scales in the evolution of infectious disease life histories: application. Evolution 65:3298-3310.

Miller, M. R., A. White, and M. Boots. 2005. The evolution of host resistance: tolerance and control as distinct strategies. Journal of Theoretical Biology 236:198-207.

- 2006. The evolution of parasites in response to tolerance in their hosts: the good, the bad, and apparent commensalism. Evolution 60:945-956.

Milner, J. M., S. D. Albon, A. W. Illius, J. M. Pemberton, and T. H. Clutton-Brock. 1999. Repeated selection of morphometric traits in the Soay sheep on St Kilda. Journal of Animal Ecology 68:472488.

Morrissey, M. B., D. J. Parker, P. Korsten, J. M. Pemberton, L. E. B. Kruuk, and A. J. Wilson. 2012. The prediction of adaptive evolution: empirical application of the secondary theorem of selection and comparison to the breeder's equation. Evolution 66:23992410 .

Morrissey, M. B., and A. J. Wilson. 2010. PEDANTICS: an R package for pedigree-based genetic simulation and pedigree manipulation, characterization and viewing. Molecular Ecology Resources 10: 711-719.

Murphy, L., P. D. Eckersall, S. C. Bishop, J. J. Pettit, J. F. Huntley, R. Burchmore, and M. J. Stear. 2010. Genetic variation among lambs in peripheral IgE activity against the larval stages of Teladorsagia circumcincta. Parasitology 137:1249-1260.

Nakagawa, S., and H. Schielzeth. 2013. A general and simple method for obtaining R2 from generalized linear mixed-effects models. Methods in Ecology and Evolution 4:133-142.

Nisbet, A. J., T. N. McNeilly, L. A. Wildblood, A. A. Morrison, D. J. Bartley, Y. Bartley, C. Longhi, I. J. McKendrick, J. Palarea-Albaladejo, and J. B. Matthews. 2013. Successful immunization against a parasitic nematode by vaccination with recombinant proteins. Vaccine 31:4017-4023.

Norris, K., and M. R. Evans. 2000. Ecological immunology: life history trade-offs and immune defense in birds. Behavioral Ecology 11:19-26.

Nussey, D. H., K. A. Watt, A. Clark, J. G. Pilkington, J. M. Pemberton, A. L. Graham, and T. N. McNeilly. 2014. Multivariate immune defences and fitness in the wild: complex but ecologically important associations among plasma antibodies, health and survival. Proceedings of the Royal Society B: Biological Sciences 281: 20132931.

Nussey, D. H., A. J. Wilson, and J. E. Brommer. 2007. The evolutionary ecology of individual phenotypic plasticity in wild populations. Lournal of Evolutionary Biology 20:831-844.

Paterson, S., K. Wilson, and J. M. Pemberton. 1998. Major histocompatibility complex variation associated with juvenile survival and parasite resistance in a large unmanaged ungulate population. Proceedings of the National Academy of Sciences of the USA 95: 3714-3719.

Pedersen, A. B., and S. A. Babayan. 2011. Wild immunology. Molecular Ecology 20:872-880.

Piedrafita, D., H. W. Raadsma, J. Gonzalez, and E. Meeusen. 2010. Increased production through parasite control: can ancient breeds of sheep teach us new lessons? Trends in Parasitology 26:568-573.

Pitala, N., L. Gustafsson, J. Sendecka, and J. E. Brommer. 2007. Nestling immune response to phytohaemagglutinin is not heritable in collared flycatchers. Biology Letters 3:418-421.

Preston, B. T., I. R. Stevenson, J. M. Pemberton, D. W. Coltman, 
and K. Wilson. 2003. Overt and covert competition in a promiscuous mammal: the importance of weaponry and testes size to male reproductive success. Proceedings of the Roval Society B: Biological Sciences 270:633-640.

Råberg, L., A. L. Graham, and A. F. Read. 2009. Decomposing health: tolerance and resistance to parasites in animals. Philosophical Transactions of the Roval Society B: Biological Sciences 364:3749.

Råberg, L., D. Sim, and A. F. Read. 2007. Disentangling genetic variation for resistance and tolerance to infectious diseases in animals. Science 318:812-814.

Restif, O., and J. C. Koella. 2003. Shared control of epidemiological traits in a coevolutionary model of host-parasite interactions. American Naturalist 161:827-836.

. 2004. Concurrent evolution of resistance and tolerance to pathogens. American Naturalist 164:E90-E102.

Robinson, M. R., A. J. Wilson, J. G. Pilkington, T. H. Clutton-Brock, J. M. Pemberton, and L. E. B. Kruuk. 2009. The impact of environmental heterogeneity on genetic architecture in a wild population of Soay sheep. Genetics 181:1639-1648.

Rohr, J. R., T. R. Raffel, and C. A. Hill. 2010. Developmental variation in resistance and tolerance in a multi-host-parasite system. Functional Ecology 24:1110-1121.

Roy, B. A., and J. W. Kirchner. 2000. Evolutionary dynamics of pathogen resistance and tolerance. Evolution 54:51-63.

Sayers, G., and T. Sweeney. 2005. Gastrointestinal nematode infection in sheep-a review of the alternatives to anthelmintics in parasite control. Animal Health Research Reviews 6:159-171.

Shaw, R. J., C. A. Morris, M. Wheeler, M. Tate, and I. A. Sutherland. 2012. Salivary IgA: a suitable measure of immunity to gastrointestinal nematodes in sheep. Veterinary Parasitology 186:109-117.

Sheldon, B. C., and S. Verhulst. 1996. Ecological immunology: costly parasite defences and trade-offs in evolutionary ecology. Trends in Ecology and Evolution 11:317-321.

Simms, E. L. 2000. Defining tolerance as a norm of reaction. Evolutionary Ecology 14:563-570.

Smith, J. A., K. Wilson, J. G. Pilkington, and J. M. Pemberton. 1999. Heritable variation in resistance to gastro-intestinal nematodes in an unmanaged mammal population. Proceedings of the Roval Society B: Biological Sciences 266:1283-1290.

Stear, M. J., K. Bairden, J. L. Duncan, P. H. Holmes, Q. A. McKellar, M. Park, S. Strain, M. Murray, S. C. Bishop, and G. Gettinby. 1997. How hosts control worms. Nature 389:27.

Stear, M. J., S. C. Bishop, M. Doligalska, J. L. Duncan, P. H. Holmes,
J. Irvine, L. McCririe, Q. A. McKellar, E. Sinski, and M. Murray. 1995. Regulation of egg production, worm burden, worm length and worm fecundity by host responses in sheep infected with Ostertagia circumcincta. Parasite Immunology 17:643-652.

Stjernman, M., L. Råberg, and J.-^̊. Nilsson. 2008. Maximum host survival at intermediate parasite infection intensities. PLoS ONE 3:e2463.

Strain, S. A., S. C. Bishop, N. G. Henderson, A. Kerr, Q. A. McKellar, S. Mitchell, and M. J. Stear. 2002. The genetic control of IgA activity against Teladorsagia circumcincta and its association with parasite resistance in naturally infected sheep. Parasitology 124:545-552.

Tobler, U., and B. R. Schmidt. 2010. Within- and among-population variation in chytridiomycosis-induced mortality in the toad Alytes obstetricans. PLoS ONE 5:e2463.

van de Pol, M. 2012. Quantifying individual variation in reaction norms: how study design affects the accuracy, precision and power of random regression models. Methods in Ecology and Evolution 3:268-280.

Williams, A. R. 2012. Short communication: some observations on the role of bradykinin in immunity to Teladorsagia circumcincta in sheep. Journal of Parasitology Research 2012:569287.

Williams, A. R., D. G. Palmer, I. H. Williams, P. E. Vercoe, and L. J. E. Karlsson. 2010. Faecal dry matter, inflammatory cells and antibodies in parasite-resistant sheep challenged with either Trichostrongylus colubriformis or Teladorsagia circumcincta. Veterinary Parasitology 170:230-237.

Wilson, A. J., D. H. Nussey, J. M. Pemberton, J. G. Pilkington, A. Morris, F. Pelletier, T. H. Clutton-Brock, and L. E. B. Kruuk. 2007. Evidence for a genetic basis of aging in two wild vertebrate populations. Current Biology 17:2136-2142.

Wilson, A. J., J. M. Pemberton, J. G. Pilkington, T. H. Clutton-Brock, D. W. Coltman, and L. E. B. Kruuk. 2007. Quantitative genetics of growth and cryptic evolution of body size in an island population. Evolutionary Ecology 21:337-356.

Wilson, A. J., D. Reale, M. N. Clements, M. M. Morrissey, E. Postma, C. A. Walling, L. E. B. Kruuk, and D. H. Nussey. 2010. An ecologist's guide to the animal model. Journal of Animal Ecology 79: 13-26.

Wilson, K., B. T. Grenfell, J. G. Pilkington, H. E. G. Boyd, F. M. D. Gulland, T. H. Clutton-Brock, and J. M. Pemberton. 2004. Parasites and their impact. Pages 113-165 in T. H. Clutton-Brock and J. M. Pemberton, eds. Soay sheep: dynamics and selection in an island population. Cambridge University Press, Cambridge.

Symposium Editor: Curtis M. Lively 